\title{
Design of a Multi-Agent System for Distributed Voltage Regulation
}

\author{
Minjiang Chen, Dimitrios Athanasiadis, Badr Al \\ Faiya, Stephen McArthur, Ivana Kockar \\ Department of Electronic and Electrical Engineering \\ University of Strathclyde \\ Glasgow, UK \\ \{minjiang.chen, dimitrios.athanasiadis, badr.alfaiya, \\ s.mcarthur, ivana.kockar\}@ strath.ac.uk
}

\author{
Haowei Lu, Francisco de Leon \\ Department of Electrical and Computer Engineering \\ New York University \\ New York, USA \\ $\{$ haowei.lu, fdeleon $\} @$ nyu.edu
}

\begin{abstract}
In this paper, an intelligent distributed multi-agent system (MAS) is proposed for the implementation of a novel optimization technique for distributed voltage regulation. The proposed MAS approach controls a large heavily-meshed distribution network which is grouped into small subnetworks using $\varepsilon$ decomposition. The voltage regulation is accomplished by distributed generator (DG) agents, linear programming solver (LPS) agents, network violation detector (NVD) agents, and one $\varepsilon$ decomposition agent. The LPS agent has an embedded control algorithm which optimizes DG generation within a subnetwork once the voltage at particular nodes exceeds the normal operational limits. The subnetworks and their control requirements are achieved through self-organization, which is the novelty of the research. Each intelligent agent has its own knowledge and reasoning logic to plan its own activities. The control actions are coordinated through agent communications within the subnetwork. The agent platform, Presage2, with improved autonomy and agent communication capability, has been used to develop the proposed MAS system and design the agents' behaviors.
\end{abstract}

Keywords-Multi-agent system; distributed generation; optimal distributed voltage regulation; $\varepsilon$ decomposition; Presage2.

\section{INTRODUCTION}

In the last few years, the connection of small and distributed generation units to the power distribution network has increased. However, these networks are traditionally designed to only have loads connected. Network control challenges are introduced when integrating distributed generation [1-4].

One of the main challenges is voltage regulation of distribution networks with DGs [5], [6]. A number of publications have tackled distributed voltage control [5-9]. A distributed control method with less information and communication requirements is introduced in [7] to mitigate overvoltage issues due to high DGs penetration. The control, performed almost as well as the optimal power flow based solution. Integrating the sensitivity methods without the aid of communication systems for a coordinated decentralized control scheme have been introduced in [9]. In [10], a multi-agent based voltage support scheme is proposed for dispatching DGs.
Another aspect of the control and computational challenges is the significant volume of data and the need to exchange information to reach a desired global performance. Hence, there is the need to investigate the advantage of distributed computational techniques, such as MAS, which may require reduced communication. In MAS, intelligent agents interact with each other to cooperatively solve network-wide problems subject to local/global constraints. From a power distribution point of view, the aim is to build local controller and decisionmaking agents that have access to local information only to solve network-wide multi-objective optimization tasks [10-13].

MAS technology has received significant scientific interest in recent years in different fields of research, such as in power systems [10], [14-19]. Some practical applications of multiagent systems in the power industry and some of the key implementations are investigated in [16]. A distributed negotiation algorithm for a distributed power system architecture is presented in [17] for distributed control of frequency and voltage using a MAS platform. An optimal decentralized voltage control scheme based on a MAS is proposed in [18] by adjusting tap-changing controllers in distribution networks.

The need for global communication systems limits the benefits of coordinated control to small distributed systems. Additionally, controlling all node voltages creates a heavy computational burden on large systems [8]. An optimal voltage regulation is proposed in [8] using $\varepsilon$ decomposition of the sensitivity matrix (inverse of the Jacobian matrix of the Newton-Raphson power flow) to group the network into subnetworks in which DGs and their influence on voltage is obtained. Based on this approach, this paper proposes a distributed MAS technique where agents within the same subnetwork are communicating to coordinate their voltage control actions.

After sub-networks are created by the application of the $\varepsilon$ decomposition method to the sensitivity matrix, each agent has its own knowledge about the network state and the information received from other agents through messages. Grouping of subnetworks varies from one decomposition value to another. Therefore, the number of agents communicating will vary to adapt to the decomposition value changes and, therefore, to minimize the communication and computation requirements. 
In this paper, Section II describes the research on the distributed voltage regulation on secondary networks with DGs. The proposed MAS framework with details of agent functions and behaviors and the simulation platform are defined in Section III. To demonstrate the agent interactions within a voltage violation scenario, agent communication examples are presented in Section IV. Section V states the conclusion of the paper and outlines the key future work.

\section{EPSILON DECOMPOSITION RESEARCH}

$\mathrm{Yu}$ et al. proposed an algorithm for the optimal voltage regulation of distribution networks with distributed generators [8]. The main idea is based on the decomposition of the sensitivity matrices obtained by performing a load flow analysis. After the load flow analysis, from the Jacobian matrices, the sensitivity matrices can be obtained which contain the relationship between changes of DG and voltage changes. Furthermore, the $\varepsilon$ decomposition [20], is used to divide the network into small subnetworks. By performing the decomposition, the strong couplings between the DGs are kept and the weak couplings are abandoned. Moreover, DGs are able to communicate with DGs that are in the same subnetwork and therefore they can control the network voltage.

The proposed voltage regulation is suitable for both power factor mode (PFC) and unity power factor (UPF) mode. The main objective is to maintain the voltage between the operational limits ( 0.95 to $1.05 \mathrm{pu})$. In the case of a violation (more than 5\%), the voltage regulation is achieved by running a linear programming optimisation problem where each DG will optimise its generation output.

This methodology was applied in a meshed distribution secondary network which has 2083 nodes (1043 nodes at 13.8 $\mathrm{kV}$ at primary feeders, the remaining 1040 nodes are at $480 \mathrm{~V}$ or $216 \mathrm{~V}$ composing the secondary network), $311 \mathrm{PQ}$ loads, and 224 network transformers $(13.8 \mathrm{kV}$ to $216 \mathrm{~V}$ or $480 \mathrm{~V})$. By applying the decomposition on the sensitivity matrix of the network the information on a DG's influence range and grouping can be obtained. The secondary network contains 19 subnetworks which are isolated.

Based on the value of the $\varepsilon$ decomposition $(0.004,0.006$, $0.008,0.01,0.012,0.014,0.016)$ a different number of DG groups, the percentage of covered nodes and a maximum number of covered nodes in one group are obtained. Additionally, for each decomposition value, there is a different successful control rate for voltage violations and also different power losses. Tables I and II provide the results of each decomposition value in both PFC and UPF mode, as reported by Yu et al. [8].

According to the tables, it can be noticed that with a larger value of $\varepsilon$ decomposition the network is divided into more groups. If the value of 0.012 is picked the network is decomposed into 82 DG groups with a maximum of 239 nodes contained in one group. This is only $62.2 \%$ of all the network nodes. However, if a smaller value is picked (0.004) then the numbers are changed and the number of groups is only 19. This means that a larger value of $\varepsilon$ decomposition covers fewer nodes of the network and more couplings are neglected. Also, the communication requirements reduced and the linear programming optimisation is able to solve the violations with a better success rate.

Voltage control simulations were performed for each load with DG under different $\varepsilon$. The simulation results show 78 voltage violations occur that need to be regulated by DGs. Moreover, a larger value has a less successful rate of solving the violation because it involves less DGs and nodes in order to find a solution.

As mentioned previously, the voltage control problem was solved by applying a linear programming optimisation method. The main idea behind this algorithm is that it is able to decrease the size of the problem for a meshed network that

TABLE I. RESULTS OF DECOMPOSITION VALUES

\begin{tabular}{|c|c|c|c|c|c|c|}
\hline \multirow{2}{*}{$\varepsilon$} & \multicolumn{3}{|c|}{ DG with PFC mode } & \multicolumn{3}{c|}{ DG with UPF mode } \\
\cline { 2 - 7 } & No. of DG groups & $\begin{array}{c}\text { \% of nodes covered in } \\
\text { DG groups }\end{array}$ & $\begin{array}{c}\text { Max. No. of nodes } \\
\text { covered in one group }\end{array}$ & No. of DG groups & $\begin{array}{c}\text { \% of nodes covered in } \\
\text { DG groups }\end{array}$ & $\begin{array}{c}\text { Max. No. of nodes } \\
\text { covered in one group }\end{array}$ \\
\hline 0.004 & 19 & 100 & 917 & 20 & 80.6 & 769 \\
\hline 0.006 & 24 & 97.7 & 888 & 35 & 61.3 & 456 \\
\hline 0.008 & 34 & 85.9 & 596 & 47 & 54.3 & 382 \\
\hline 0.01 & 57 & 74.6 & 412 & 75 & 46.7 & 142 \\
\hline 0.012 & 82 & 62.2 & 239 & 72 & 37.6 & 58 \\
\hline 0.014 & 80 & 52.0 & 163 & 75 & 34.5 & 48 \\
\hline 0.016 & 85 & 40.8 & 47 & & & 48 \\
\hline
\end{tabular}

TABLE II. RESULTS OF VOLTAGE CONTROL SIMULATIONS FOR DG IN PFC MODE

\begin{tabular}{|c|c|c|c|c|c|c|c|c|}
\hline \multirow{2}{*}{$\begin{array}{c}\text { Successful } \\
\text { control rate }\end{array}$} & \multirow{2}{*}{$\begin{array}{c}\text { No. of groups with } \\
\text { under-voltage }\end{array}$} & \multirow{2}{*}{$\begin{array}{c}\text { Average No. of } \\
\text { nodes involved }\end{array}$} & \multirow{2}{*}{$\begin{array}{c}\text { Average No. of } \\
\text { DGs involved }\end{array}$} & \multicolumn{2}{|c|}{$\begin{array}{c}\text { Average power loss before } \\
\text { control (p.u.) }\end{array}$} & \multicolumn{2}{|c|}{$\begin{array}{c}\text { Average power loss after control } \\
\text { (p.u.) }\end{array}$} \\
\cline { 7 - 10 } & & & & & Active power & Reactive power & Active power & Reactive power \\
\hline 0.004 & $76 / 78$ & 1 & 131.4 & 21.3 & 0.2937 & 0.8551 & 0.3536 & 0.9135 \\
\hline 0.006 & $75 / 78$ & 1 & 67.9 & 15.3 & 0.2938 & 0.8551 & 0.3504 & 0.9078 \\
\hline 0.008 & $74 / 78$ & 2 & 42.3 & 12.4 & 0.2943 & 0.8555 & 0.3516 & 0.9063 \\
\hline 0.010 & $71 / 78$ & 5 & 31.2 & 10.4 & 0.2952 & 0.8559 & 0.3534 & 0.9042 \\
\hline 0.012 & $71 / 78$ & 12 & 23.4 & 8.6 & 0.2914 & 0.8440 & 0.3493 & 0.8901 \\
\hline 0.014 & $70 / 78$ & 15 & 18.7 & 7.1 & 0.2956 & 0.8561 & 0.3524 & 0.8984 \\
\hline 0.016 & $69 / 78$ & 25 & 14.9 & 6.1 & 0.2956 & 0.8562 & 0.3527 & 0.8976 \\
\hline
\end{tabular}


contains more than 100 constraints to almost 12. This algorithm is able to find the optimal solution for each decomposition value and also can regulate the voltage from the involved DGs.

The proposed methodology can be performed by multiagent systems where control and measurement agents will be installed in the network. Based on the decomposition values the communication links between the agents will be determined. The next section will analyse the environment of the agents and will provide a methodology to integrate the $\varepsilon$ decomposition algorithm into a multi-agent systems environment.

\section{Design OF THE Multi-AGENT SYSTEM FOR DiSTRIBUTED VOLTAGE REGULATION}

The voltage regulation approach proposed decomposes the network voltage regulation problem into several subnetwork voltage regulation problems, based on the $\varepsilon$ decomposition. In order to deploy this voltage regulation method within a MAS framework, the MAS framework contains an $\varepsilon$ decomposition agent, a linear programming solver (LPS) agent, a network violation detection (NVD) agent and a DG agent. After the large network is divided into a number of subnetworks by using the $\varepsilon$ decomposition, each subnetwork has one LPS agent, one NVD agent and one or more DG agents. The number of LPS agents and NVD agents varies from value to value and must be the same as the subnetwork numbers. In order to do that, $\varepsilon$ decomposing agent is the one to generate or kill the LPS agent and NVD agent according to the decomposition result. Furthermore, each agent can plan its own activities by using its knowledge and behaviors. Agents can communicate and cooperate with each other to realize the voltage regulation using an agent communication language (ACL). The knowledge and behaviors within the $\varepsilon$ decomposition agent, DG agent, LPS agent and NVD agent are detailed below.

\section{A. $\varepsilon$ Decomposition Agent}

The $\varepsilon$ decomposition agent has knowledge of the $\varepsilon$ decomposition value. Moreover, the $\varepsilon$ decomposition agent will update the $\varepsilon$ decomposition value according to its knowledge, which could be nodes changing (e.g. adding a new DG) or failing to solve the voltage violation. If there is a change in the nodes of the network or a failure to solve the voltage violation, the $\varepsilon$ decomposition agent will select a new value and create a new sensitivity matrix that will reset the subnetworks and zones of influence of each DG. After that, the $\varepsilon$ decomposition agent will inform DG agents once it determines a new $\varepsilon$ decomposition value. Then, the DG agents will re-group and self-organise without a complete reengineering of the overall MAS framework. As a result, the MAS platform and other agents do not need to be stopped or restarted, and this can happen at any point. As mentioned above, the $\varepsilon$ decomposition agent is responsible for creating the NVD, and LPS agents based on the number of subnetworks Therefore, the $\varepsilon$ decomposition agent will group LPS and NVD agents with the subnetwork number and will assign their names and addresses for each subnetwork. Then, the $\varepsilon$ decomposition agent informs each LPS agent about which DG agents are in the same subnetwork, providing the names and addresses to ensure the LPS agent links with the DG agents within the same subnetwork. The same approach is taken for the $\varepsilon$ decomposition agent to inform the NVD agent about which LPS agent is in the same subnetwork.

\section{B. Distributed Generator(DG) Agent}

The DG agent takes action to control the DG after receiving a command from the LPS. In addition, the DG agent has knowledge of the sensitivity matrix so that it will dynamically update its voltage influence range and, that of other DGs in the same group, based on different $\varepsilon$ decomposition values.

\section{Linear Programming Solver (LPS) Agent}

The LPS agent is responsible for solving the voltage violation within the subnetwork by means of a linear programming (LP) algorithm. In order to do that, each subnetwork has one LPS agent to regulate the voltage within the subnetwork. Furthermore, the LP algorithm is integrated within a LPS agent, therefore it requires knowledge of the sensitivity matrix to determine the appropriate DGs following notification of nodes where violations have occurred. Once it receives a violation message it seeks a solution by employing the LP algorithm. After determining solutions for using the appropriate DG's output, the LPS agent sends the control value to the DG agent(s) to take action through agent messaging. If the LPS does not find a solution when a voltage violation occurs within the subnetwork, it will send a message to the $\varepsilon$ decomposition agent about failing to find solution so that the $C$ decomposition agent may select a smaller epsilon value to cover more DGs in order to regulate the violation.

\section{Network Violation Detector (NVD) Ag ent}

The role of the NVD agent is to monitor the status of its subnetwork, and to identify events such as voltage violations. Therefore, each subnetwork has one NVD agent. Additionally, the NVD agent has knowledge of the voltage operation limit, which is between 0.95 p.u. and 1.05 p.u. for this research. Once the NVD agent detects a voltage violation within its subnetwork, it communicates with its subnetwork's LPS agent with the violation nodes to be solved. If the solution is not successful following the LPS agent sending it to the DG agents and the application of the control actions, the NVD agent informs the $\varepsilon$ decomposition agent to choose a smaller epsilon value so that more nodes are covered and more DGs are involved in order to alleviate that node's violation.

\section{E. Agent Simulation Platform}

The proposed MAS is being implemented within the simulation of agent societies 2 (Presage2) framework [21-22]. Presage is a JAVA based programming environment that provides improved autonomy and agent communication capability. Presage 2 contains abstract classes and interfaces for the user to extend. It allows for the trialing of MAS prototypes and the design of novel advanced applications in electric power systems. In addition, Presage 2 offers the flexibility to design advanced self-organising systems. Furthermore, the Presage2 agent framework has already been used to build a MAS system 
for flexible network management, which integrates various control algorithms, such as distributed negotiation [17] and power flow management [23].

The MAS simulation environment is shown in Fig. 1. In the MAS platform environment, agents collaborate with each other by agent communications through the agent message transport system. The agent message transport system is the central switchboard for messaging in the platform, rather than letting the agents communicate with each other directly. Users can add constraints to allow the blocking of messages or modify the messages before delivery. Presage 2 has defined its own ACL. The message format in Presage 2 is:

\section{Message (Performative, Sender, Receiver, Time, Content)}

The Performative indicates the intention behind the message, such as "require", "query" and "inform" that are defined in the FIPA-ACL standard [24]. The Sender and Receiver refer to the agents that are sending and receiving the message respectively. Time of the message is the time at which the message was sent. The Content is the information that is communicated between the agents. Hence, Presage 2 provides the essential infrastructure for agent design, simulation, and communications.

\section{F. Agent Architecture within Presage2}

Each agent has social ability and functions to achieve their delegated task. The design of an LPS agent is illustrated in Fig. 2 as an example. Each agent has a message box to receive and send messages such as voltage violation messages or DG control action messages. In addition, each agent exhibits various behaviors. For instance, the LPS agent has a solution behavior, which is to find the optimal output of the appropriate DG(s) to solve the voltage violation within the subnetwork and then inform the DG agents to take action. The NVD agent's behavior, on the other hand, is to detect the violation within its subnetwork and to inform the corresponding LPS agent once a violation occurs. Moreover, the knowledge of the LPS agent shown in Fig. 2 includes the $\varepsilon$ decomposition and sensitivity matrix.

\section{MAS IMPLEMENTATION FOR DISTRIBUTED VOLTAGE REGULATION}

In this section, the agent interaction to achieve the distributed voltage regulation will be illustrated. The agent interaction describes how each agent communicates at a different stage to coordinate with other agents, based on its behaviors and knowledge, to control the voltage in a distributed manner.

\section{A. MAS Implementation Overview}

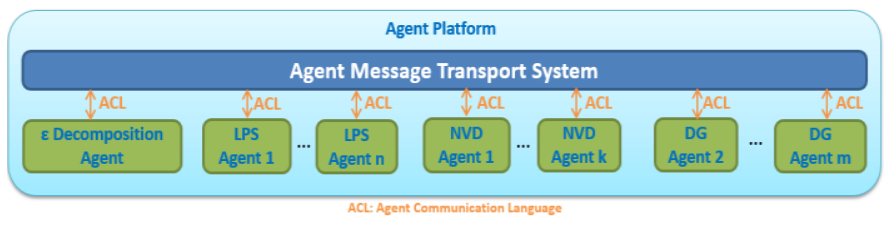

Fig. 1. MAS for optimal distributed voltage regulation within Presage2.
The procedure to implement the proposed MAS is discussed below.

Step 1: When initializing the system, the decomposition agent selects an initial $\varepsilon$ decomposition value. This value is based on the purpose of the decomposition, i.e. the desire to have more distributed control, less communication and less computation within each subnetwork. The $\varepsilon$ decomposition value should start with the highest value that results in the largest number of subnetworks.

Step 2: To launch the system, the $\varepsilon$ decomposition agent calculates the initial sensitivity matrix with the initial $\varepsilon$ decomposition value. Then, the $\varepsilon$ decomposition agent generates the initial number of LPS agents and NVD agents according to the subnetworks identified. Next, the $\varepsilon$ decomposition agent informs LPS agents and NVD agents about their subnetwork agents to allow them to link with each other. Moreover, the $\varepsilon$ decomposition agent informs the DG agents about the $\varepsilon$ decomposition value so they know which subnetwork they belong to. After that, each LPS agent, NVD agent and DG agent loads the sensitivity matrix and decomposition result to ensure they have appropriate knowledge, such as subnetwork groups and each DG's voltage influence range.

Step 3: Each NVD agent starts to check for voltage violations on its subnetwork. If there is a voltage violation, the NVD agent sends a violation message with violation nodes to its subnetwork LPS agent. The LPS agent checks the violation message and determines the involved DG(s) within the subnetwork to optimize each DG's output. After identifying the solution, the LPS agent sends control actions to the related DG agent(s).

Step 4: If the LPS agent cannot find a solution it sends feedback to the decomposition agent in order to pick a smaller $\varepsilon$ decomposition value. However, if the LPS agent finds a solution and the NVD agent still detects a violation, the NVD agent is responsible for sending a message to the $\varepsilon$ decomposition agent to select another value to solve the violation. When the $\varepsilon$ decomposition agent determines a new $\varepsilon$ decomposition value it calculates the new sensitivity matrix and re-generates the subnetworks and zones of influence based on this. Moreover, the $\varepsilon$ decomposition agent will kill the LPS agent(s) and NVD agent(s) to generate new LPS agent(s) and NVD agent(s). After that, it will move to Step3 to continue the simulation.

An example of this agent self-organisation and co-operation for voltage regulation (under the different $\varepsilon$ decomposition values) is presented below.

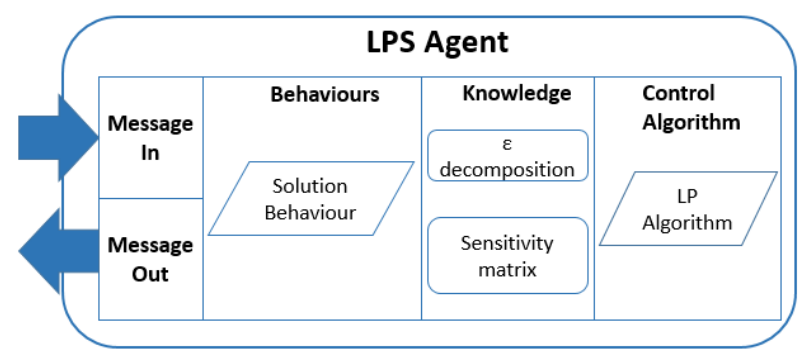

Fig. 2. LPS agent architecure within Presage2. 


\section{B. Agents Interaction with Voltage Regulation}

According to the initial $\varepsilon$ decomposition value, a large secondary distribution network can be grouped into several subnetworks. The initial $\varepsilon$ decomposition result is depicted schematically in Fig. 3. The distributed network has been isolated into 5 subnetworks. As a result, the group of DGs and the range of influence of each DG on the voltage of its subnetwork have been determined. Each subnetwork contains one LPS agent, one NVD agent and one or more DG agents. As the distributed network breaks into small isolated subnetworks, each agent only needs to communicate with other agents that are in the same subnetwork.

After the initial decomposition, the NVD agent begins to monitor for voltage violations on its subnetwork. The agent communication diagram for the simulation is portrayed in Fig. 4. As shown in the agent communication diagram, the NVD agent 1 observed that there was a voltage violation within its subnetwork. Then, NVD agent 1 sends a voltage violation message to the LPS agent 1 to solve the voltage regulation problem. Once the LPS agent 1 found a control solution, it sends the optimized output to the DG agent 1 (as DG agent 1 is the involved DG in this case) to take action to adjust the output of the DG 1. After that, another voltage violation occurred within the subnetwork 5 . Then the NVD agent 4 informs the LPS agent 5 about a voltage violation need to be solved. However, LPS agent 5 did not find a solution and therefore it informs the $\varepsilon$ decomposition agent about failing to find a solution.

As a result, the $\varepsilon$ decomposition agent selects a new value.

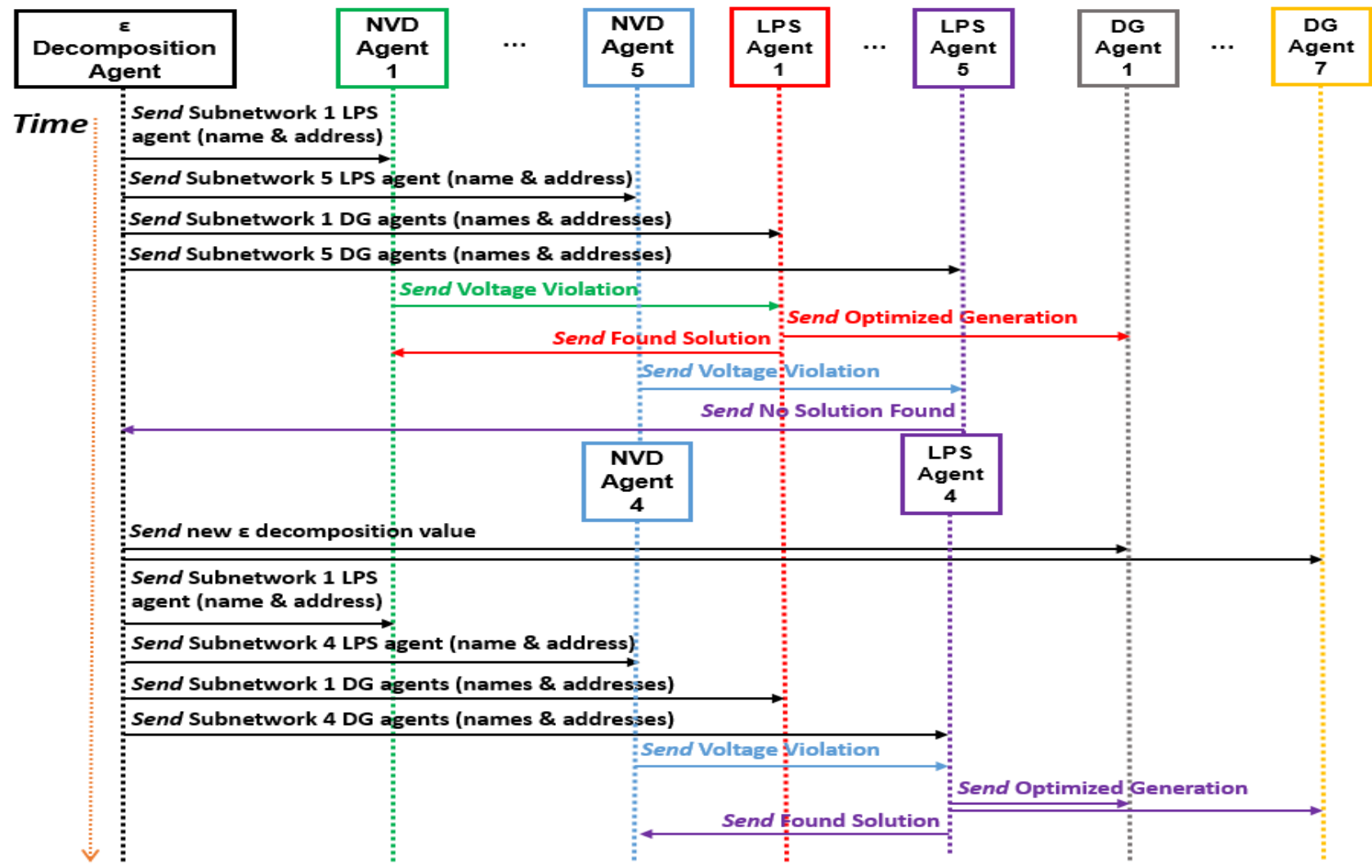

Fig. 4. Agent's interactions for voltage regulation.



Fig. 3. Initial $\varepsilon$ decomposition result.

The new decomposition result is shown in Fig. 5. The number of the subnetworks is changed to 4 . Hence, $\varepsilon$ decomposition agent kills the old NVD agents or LPS agents and creates new NVD agents and LPS agents. Therefore, subnetwork 4 covers more DGs by comparison with the initial decomposition result. NVD agent 4 detected the same voltage violation within subnetwork 4 and informed the related LPS agent 4 to mitigate the violation as demonstrated in the agent communication diagram in Fig. 4. Finally, the solution message is sent to DG agent 1 and 7 (as DG agent 1 and 7 are the appropriate DGs in this case) to take actions after the optimal adjustment determined by LPS agent 1 . 


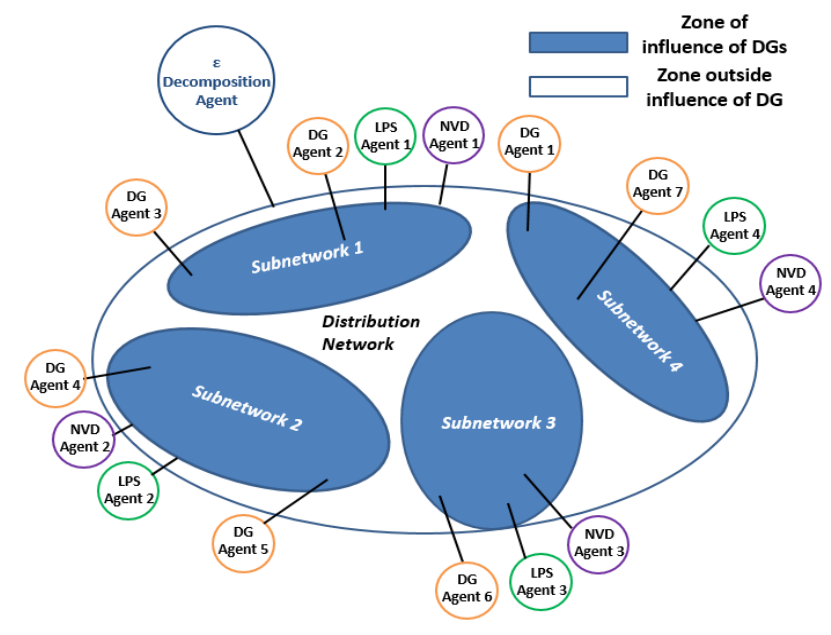

Fig. 5. Second $\varepsilon$ decomposition result.

\section{CONCLUSION AND FUTURE WORK}

This paper described a novel MAS for voltage regulation within a distributed network. Using the $\varepsilon$ decomposition value, the large secondary distributed network is divided into several subnetworks. Moreover, this effectively decomposes the network voltage regulation into several subnetwork voltage regulation problems. Hence, control coordination using system communications can be achieved within the subnetwork as well. In order to achieve this, the proposed MAS framework is composed of an $\varepsilon$ decomposition agent, a NVD agent, a DG agent and an LPS agent. Each subnetwork has one NVD agent, one LPS agent and one or more DG agents coordinating with each other to regulate voltage violation. The proposed $\varepsilon$ decomposition agent can update the new $\varepsilon$ decomposition value based on its feedback from subnetworks. As a result, the DG agent, LPS agent and NVD agent update their knowledge of a DG's influence on voltage and which subnetwork it belongs to. Furthermore, the LPS agents and NVD agents can be created or killed according to the number of subnetworks without re-engineering of the complete system. This MAS approach and design offers novel self-organization capabilities. For the next step, the MAS framework will be tested with a model of a real heavily meshed secondary network. It will also determine how to choose the $\varepsilon$ decomposition value for the system decomposition.

\section{REFERENCES}

[1] E. J. Coster, J. M. A. Myrzik, B. Kruimer, and W. L. Kling, "Integration issues of distributed generation in distribution grids," Proceedings of the IEEE, vol. 99, no. 1, pp. 28-39, Jan. 2011.

[2] M. Cavlovic , "Challenges of optimizing the integration of distributed generation into the distribution network," 2011 8th International Conference on the European, Zagreb, 2011.

[3] H. Farhangi, "The Path of the Smart Grid," in IEEE Power and Energy Magazine, vol. 8, no. 1, pp. 18-28, January-February 2010.

[4] T. Sansawatt, L. F. Ochoa, and G. P. Harrison, "Integrating distributed generation using decentralised voltage regulation," Power and Energy Society General Meeting, 2010 IEEE. pp. 1-6, 2010.

[5] P.P. Barker, R.W. De Mello, "Determining the impact of distributed generation on power systems. I. Radial distribution systems," Power Engineering Society Summer Meeting, 2000. IEEE, vol.3, no., pp.16451656 vol. 3, 2000.
[6] L. Martini, L. Radaelli, H. Brunner, C. Caerts, A. Morch, S. Hanninen, and C. Tornelli. "ELECTRA IRP approach to voltage and frequency control for future power systems with high DER penetration." In Proceedings of 23rd International Conference on Electricity Distribution (CIRED), Lyon. 2015.

[7] F. Olivier, P. Aristidou, D. Ernst, and T. Van Cutsem, "Active Management of Low-Voltage Networks for Mitigating Overvoltages Due to Photovoltaic Units," in IEEE Transactions on Smart Grid, vol. 7, no. 2, pp. 926-936, March 2016.

[8] Y. Li, D. Czarkowski, and F. De León. "Optimal distributed voltage regulation for secondary networks with DGs." IEEE Transactions on Smart Grid, vol. 3, no. 2, pp. 959-967, Jun. 2012.

[9] A. Samadi, R. Eriksson, L. Söder, B. G. Rawn, and J. C. Boemer, "Coordinated Active Power-Dependent Voltage Regulation in Distribution Grids With PV Systems," in IEEE Transactions on Power Delivery, vol. 29, no. 3, pp. 1454-1464, June 2014.

[10] M. E. Baran and I. M. El-Markabi, "A multiagent-based dispatching scheme for distributed generators for voltage support on distribution feeders," IEEE Transactions on Power Systems, vol. 22, no. 1, pp. 5259, Feb. 2007.

[11] S. D. J. McArthur, E. M. Davidson, V. M. Catterson, A. L. Dimeas, N. D. Hatziargyriou, F. Ponci, and T. Funabashi, "Multi-Agent Systems for Power Engineering Applications-Part I: Concepts, Approaches, and Technical Challenges," in IEEE Transactions on Power Systems, vol. 22, no. 4, pp. 1743-1752, Nov. 2007.

[12] S. D. J. McArthur, E. M. Davidson, V. M. Catterson, A. L. Dimeas, N. D. Hatziargyriou, F. Ponci, and T. Funabashi, "Multi-Agent Systems for Power Engineering Applications-Part II: Technologies, Standards, and Tools for Building Multi-agent Systems," in IEEE Transactions on Power Systems, vol. 22, no. 4, pp. 1753-1759, Nov. 2007.

[13] D. E. Olivares et al., "Trends in Microgrid Control," in IEEE Transactions on Smart Grid, vol. 5, no. 4, pp. 1905-1919, July 2014.

[14] F. Z. Harmouch, N. Krami, D. Benhaddou, N. Hmina, E. Zayer, and E. H. Margoum, "Survey of multiagents systems application in Microgrids," 2016 International Conference on Electrical and Information Technologies (ICEIT), Tangiers, 2016, pp. 270-275.

[15] A. L. Dimeas and N. D. Hatziargyriou, "Control Agents for Real Microgrids," 15th International Conference on Intelligent System Applications to Power Systems (ISAP), Curitiba, 2009, pp. 1-5.. V. M. Catterson, E. M. Davidson and S. D. McArthur, "Practical Applications of Multi-agent Systems in Electric Power Systems," European Transactions on Electrical Power, 22(2), 235-252. Aug.2011.

[16] M. Chen, M. H. Syed, E. G. Sansano, S. D. J. McArthur, G. M. Burt, and I. Kockar, "Distributed Negotiation in Future Power Networks: Rapid Prototyping Using Multi-agent System," IEEE PES Innovative Smart Grid Technologies Conference Europe (ISGT-Europe), Ljubljana, Slovenia, 2016.

[17] N. Yorino, Y. Zoka, M. Watanabe, T. Kurushima, "An Optimal Autonomous Decentralized Control Method for Voltage Control Devices by Using a Multi-Agent System", IEEE Transactions on Power Systems, 2015.

[18] S. D. J. McArthur et al., "The Autonomic Power System - Network operation and control beyond smart grids," 3rd IEEE PES Innovative Smart Grid Technologies Europe (ISGT Europe), Berlin, 2012.

[19] D. D. Šiljak, Decentralized Control of Complex Systems ser. Mathematics in Science and Engineering, USA, CA, San Diego: Academic, vol. 184, 1991.

[20] B. Neville, J. Pitt, "PRESAGE: A Programming Environment for the Simulation of Agent Societies," in Proc. 2008 AAMAS Workshop on Programming Multi-agent Systems (ProMAS).

[21] S. Macbeth, D. Busquets, and J. Pitt, "Principled operationalization of social systems using Presage2," in D. Gianni, A. D'Ambrogio, and A. Tolk, Modeling and Simulation-Based Systems Engineering Handbook, CRC Press, pp. 43- 66, 2014.

[22] M. Chen, S. D. J. McArthur, I. Kockar, and J. Pitt, "Evaluating a MAS architecture for flexible distribution power flow management," 18th International Conference on Intelligent System Application to Power Systems (ISAP), Porto, 2015

[23] FIPA [Available online]: http://www.fipa.org/repository/aclspecs.html 